\title{
Modelo Social Europeo y políticas sociales: una evaluación formativa institucional
}

\author{
Luis Moreno \\ Amparo Serrano*
}

Resumen: Las instituciones europeas ocupan un lugar importante en los procesos de reforzamiento de la dimensión social de las economías europeas. Este artículo analiza algunas de las potencialidades, pero también las limitaciones del proceso de europeización de la cuestión social que promueve el Modelo Social Europeo (MSE). Se realiza una evaluación formativa de carácter institucional de la europeización con el fin de tomar el pulso al proceso de supra-nacionalización, y a los avances y frenos que conlleva. Se presta especial atención al carácter dual y ambivalente del Modelo Social Europeo integrador de crecimiento económico y cohesión social, y que se sustenta en un sistema axiológico que valora lo social en igual medida-cuando menos-que lo económico.

Palabras clave: Agenda de Lisboa, activación laboral, gobernanza europea, Método Abierto de Coordinación (MAC), Proceso de Luxemburgo, regímenes del bienestar.

\section{INTRODUCCIÓN}

Desde hace dos décadas las instituciones comunitarias protagonizan uno de los procesos de regulación supranacional de "la cuestión social" más ambiciosos desde los orígenes del proyecto europeo. La estrategia social europea plantea intensificar la cooperación entre el nivel nacional y el europeo, con el fin de articular una estrategia coordinada de promoción del empleo y de lucha contra la exclusión social. Tras la Unión

* Luis Moreno ha trabajado en el presente texto durante el desarrollo del proyecto NURSOPOB ("Nuevos Riesgos Sociales y Trayectorias de las Políticas del Bienestar" (Plan Nacional I+D+I, Ministerio de Educación y Ciencia, SEJ2005-06599) y ha utilizado su texto elaborado para la Fundación Carolina (Moreno, 2006). Amparo Serrano ha contado para la redacción de este artículo con las conclusiones del proyecto “Evaluación cualitativa de las políticas de activación: los límites de lo activo y lo pasivo” (proyecto I+D+I del Ministerio de Educación y Ciencia, SEJ2007-64604) y del proyecto "Protección y flexiguridad. La modernización de los servicios públicos de empleo" (proyecto FIPROS 2008/35 del Ministerio de Trabajo e Inmigración). Los autores agradecen los comentarios anónimos de los evaluadores externos de esta revista. 
Económica y Monetaria (UEM), creada oficialmente en 1988, se estableció una clara asimetría entre las políticas de promoción económica, que han sido en gran medida europeizadas, y las políticas de promoción social, que siguen desarrollándose mayormente en el ámbito de los estados miembros (EE.MM.). Los procesos de globalización económica, a los que la UEM contribuye, ponen en evidencia la necesidad de reforzar la dimensión supranacional de la protección social. A su vez, con la UEM se ha reducido el margen de maniobra a disposición de los gobiernos de los EE.MM. para influir en el curso de sus economías con fines sociopolíticos (ajustar la tasa de cambio, devaluación monetaria, gasto público potencialmente deficitario, promoción pública de empleo o ayudas estatales, por citar algunas actuaciones del pasado), y para llevar a cabo políticas macroeconómicas en sus ámbitos estatales potencialmente favorables a la cohesión social (Scharpf, 2002). Además existe el riesgo de presión a la baja en los salarios, en las cargas impositivas y en el sistema de seguridad social, dada la facilidad con la que las empresas pueden trasladar (deslocalizar) el lugar de producción.

A la vista de esta situación, parece existir un cierto consenso acerca de la necesidad de reforzar el proyecto social europeo. Pero este proyecto no es evidente, dada la importante diversidad de mercados de trabajo en el interior de Europa, y por tanto, la gran variación de problemas y situaciones de las relaciones industriales en estos plurales mercados de trabajo. A su vez, nos encontramos ante una gran diversidad de tipos o "regímenes del bienestar", y por tanto, no solo una importante variación en cuanto a la capacidad de los Estados de Bienestar para hacer frente a las necesarias transferencias sociales, sino también con una variedad de valores sociales y culturales que justifican y legitiman la necesidad de estas transferencias. Además el traspaso del control de las políticas sociales a las instituciones europeas sería objeto de una gran resistencia política si ello se realizase en modo rápido y lineal. Las políticas sociales son el resultado de largas tradiciones históricas y compromisos políticos a nivel de los EE.MM., por lo que aún no conforman una identidad cultural transnacional europea. No sorprende que los EE.MM. se hayan mostrado reticentes a un traspaso hacia las instituciones de la UE de competencias con tanto simbolismo cultural como son las políticas sociales.

El proyecto europeo confronta, por tanto, una importante paradoja, cual es cómo reforzar la dimensión social a nivel supranacional en un contexto de dificultades políticas para su regulación. En este marco paradójico ha sido "inventado" el Método Abierto de Coordinación (MAC) que caracteriza, cada vez en mayor medida, al modelo social europeo. Este artículo analiza algunas de las potencialidades, pero también de los problemas, que plantean los principales instrumentos y propuestas que articulan este Modelo Social Europeo (MSE). Ello se realiza desde una perspectiva de evaluación formativa ${ }^{1}$ de carácter institucional y no es, por tanto, conclusiva

$1 \quad$ Entre los diversos tipos de evaluaciones, la formativa trata de responder a la pregunta: “¿Cómo puede mejorarse la intervención que se está llevando a cabo?”. En contraste con la conclusiva, orientada prioritariamente a enjuiciar los resultados finales, la evaluación formativa se ocupa del proceso en sí con el objetivo de calibrar las metas propuestas a la luz de los cambios que se consideran que puedan optimizar

12 las actividades en marcha (Moreno y Ziglio, 1992). 
respecto a políticas sociales concretas. No se corresponden, por tanto, nuestras reflexiones a análisis empíricos de programas o actuaciones sectoriales o integrales en el desarrollo inacabado del Modelo Social Europeo, el cual se examina con carácter general en la próxima sección. Se trata, más bien, de tomar el pulso político-institucional al proceso de europeización social. Uno de los principales instrumentos de este proceso europeizador es el Método Abierto de Coordinación (MAC), el cual se analiza en la sección tercera como instrumento de la gobernanza europea basado en la desformalización de los recursos normativos, la descentralización deliberativa y la autorregulación. Una de las principales potencialidades de este modo de regulación es su capacidad de difusión de conceptos que articulan marcos² de discusión acerca de la cuestión social a nivel nacional. Uno de los conceptos más importantes difundidos por estas instituciones es el de activación. Posteriormente, y a fin de ilustrar las potencialidades y limitaciones de este MAC, se da cuenta de la ambivalente evaluación del nuevo paradigma activador aceptado por la mayoría de países y regímenes europeos del bienestar. Tales análisis son previos a las valoraciones del Proceso de Luxemburgo y de la Agenda de Lisboa del apartado subsiguiente. Finalmente, y entre otros, los comentarios finales subrayan el carácter dual y ambivalente del Modelo Social Europeo integrador de crecimiento económico y cohesión social.

\section{2. ¿QUÉ ES EL MSE?}

En modo amplio cabe identificar al Modelo Social Europeo como un proyecto articulado en torno a la solidaridad colectiva, la equidad social y la eficiencia productiva. Los principios que delimitan el MSE contrastan con otros sistemas socio-económicos donde el individualismo re-mercantilizador es el rasgo característico de las políticas del bienestar (EE.UU.) ${ }^{3}$, o el modelo de dumping social que se propone como valor añadido de crecimiento económico (países emergentes asiáticos). EI MSE promueve la ciudadanía social entendida como una limitación a la desigualdad social y económica, una mayor protección a los más vulnerables y un partenariado social activo. Como objetivo estratégico, el MSE auspicia el crecimiento económico sostenido y sostenible basado en la cohesión social (Scharpf, 2002; Adnett y Hardy, 2005; Jepsen y Serrano Pascual, 2005; Giddens, 2006).

Al plasmar el ideal de cohesión social en la práctica de las políticas económicas y sociales, así como en la organización institucional del bienestar, surge una diversidad de matices. Para la Confederación Europea de Sindicatos, por ejemplo, el concepto de cohesión social implica una mejora de las condiciones de vida y trabajo de los ciudadanos basada en el pleno empleo, trabajos de calidad, igualdad de oportunidades,

2 En el sentido que plantea Lakoff (2007).

3 Tras analizar datos longitudinales de un conjunto de indicadores, se ha apuntado que el rango de variación dentro de la Unión Europea es mayor que entre la UE y los Estados Unidos. Sin embargo, no cabe hablar de diferentes modelos sociales europeos sino de diferentes trayectorias internas (Alber, 2006). 
protección social para todos, inserción social y participación ciudadana (ETUC, 2005). Para la visión patronal menos opuesta a la flexiseguridad, la cohesión se traduciría en una combinación de despidos más fáciles, aunque con altas prestaciones para los desempleados y una política social proactiva hacia el mercado laboral (EuroActiv, 2005). La Asamblea de las Regiones Europeas añade la igualdad de género y el acceso universal a prestaciones y servicios sociales basado en la solidaridad (AER, 2005). Naturalmente hay voces discordantes respecto a la plausibilidad de recomendar para un país europeo la adopción de un modelo comprensivo que pudiera funcionar en algunos países, pero que en otros resultaría contraproducente (Munchau, 2005). Incluso se ha aducido que el MSE está siendo gradualmente socavado por la realidad del cambio económico global4.

El MSE aparece como recurso y objetivo inherente al proceso de europeización. No parece plausible, en este sentido, especular con un escenario futuro en que lo social pudiese quedar desgajado de lo económico en la progresiva institucionalización del ámbito político europeo (Flora, 1993; Flora et al., 1999; Ferrera, 2005). Ciertamente deben rehuirse los ejercicios de ocultación respecto a las dificultades del proceso europeizador, abocado a regular en un marco de polisemia política y diversidad geográfica, y, por ello, conviene tener en cuentas las varias legitimidades del bienestar sedimentadas en el pasado y que se examinan brevemente a continuación.

\section{Europeización y regímenes del bienestar}

El proceso de europeización implica una confluencia de recursos, representaciones sociales y acciones entre los países de la Unión Europea (UE). Ello es resultado, principalmente, de la difusión de ideas y valores compartidos, de los procesos de armonización estructural económica, de la construcción de un sistema institucional transnacional y de una común preocupación por lo social. La europeización implica a países que comparten una herencia común y asumen valores democráticos de igualdad y derechos humanos. Empero, el concepto de europeización adolece de precisión normativa. Es polisémico y sujeto a diversas interpretaciones. Su naturaleza dinámica se refleja en una cierta erosión de la soberanía de los EE.MM. de la Unión Europea y en el gradual desarrollo de instituciones y políticas comunes supraestatales (Acuerdo de Schengen, Euro y UEM o Tribunal de Justicia, por ejemplo).

La constitución de unos Estados Unidos de Europa no debe ser necesariamente el resultado final de la europeización. Indudablemente el proceso europeizador se caracteriza por la emergencia de un nivel europeo de estructuras de gobernanza y

4 Se ha argüido que el modelo podría ser insostenible financieramente en un futuro a medio/largo plazo. Las tasas altas de fiscalidad podrían frenar no solo la inversión sino la creación de nuevo empleo. Considérese, por ejemplo, que los asalariados sin hijos a su cargo pagan la mitad de sus estipendios en cotizaciones sociales e impuestos en Bélgica y Alemania. En Nueva Zelanda pagan poco más del 20\%, y aún menos en economías de rentas medias como en México y Corea del Sur (Shackleton, 2006). 
por la creación de normas europeas de regulación (Cowles, Caporaso y Risse, 2001). Sin embargo, el entendimiento de la integración institucional en Europa no puede basarse en la asimilación cultural y la formación identitaria al modo del melting-pot estadounidense. A menudo esta visión funcionalista se ha asociado en modo espurio con la organización estatalista vertical y jerarquizada del "ordeno-y-mando" (command-and-control) en la organización socioeconómica del bienestar social (Moreno y McEwen, 2005).

Alternativamente, existe otro enfoque que considera que las normas europeas solo pueden legitimarse teniendo en cuenta la historia y la diversidad cultural característica del mosaico de pueblos y politeyas que configuran el “Viejo Continente”. La descentralización es un elemento crucial del proceso que confronta el desafío de optimizar la asignación de recursos y competencias sobre la base de los dos principios guía en el proceso de Europeización: subsidiariedad territorial y responsabilidad democrática (Moreno, 2003).

En los últimos lustros, los procesos de cambio y reforma en los Estados de Bienestar europeos se han acelerado condicionados por las características de sus distintas ideologías, intereses e instituciones (Ferrera, 1993; Moreno y Palier, 2005; Sapir, 2005). Recordemos que hablar de un "régimen de bienestar" denota que, en la relación entre estado, sociedad y economía, un complejo haz de rasgos legales y organizativos se encuentra entremezclado articuladamente (Esping-Andersen, 1990). Además hay una dimensión macrocomparativa que permite agrupar a los países de acuerdo a su "lógica del bienestar" 5 . Con el propósito de valorar los legados históricos y las específicas inercias institucionales (path dependencies) en la conformación futura del MSE, analizamos a continuación sintéticamente los cuatros grandes regímenes del bienestar en la UE-15 hasta el año $2004^{6}$ :

- Continental. Organizado por un sistema de seguridad social según categorías ocupacionales, su finalidad es garantizar el mantenimiento de ingresos y el estatus de los asalariados contribuyentes. Existe una provisión subsidiaria de servicios sociales por parte de los agentes sociales (sindicatos, iglesias, asociaciones profesionales). Estos adoptan prácticas corporatistas de concertación social en la producción del bienestar ciudadano que cuentan con la sanción legitimadora del estado. La universalidad en la cobertura del bienestar social depende del logro y mantenimiento del pleno empleo.

5 Naturalmente los países agrupados en "regímenes del bienestar" poseen algunas características propias y distintivas. El diseño metodológico trata básicamente de correlacionar variables, dependientes e independientes, que persiguen determinar la variabilidad o convergencia de los países (Esping Andersen, 1993 y 1999).

6 Las características de los "regímenes del bienestar" y las especificidades intra-grupales nacionales se han visto contrastadas con la integración en la UE de nuevos miembros del Este europeo (Guillén y Palier, 2004). A pesar de sus antecedentes comunes como países comunistas bajo el control de la ex-Unión Soviética, es prematuro agrupar a los países del Este europeo en un nuevo régimen distintivo del capitalismo del bienestar. En realidad se denotan características dispares entre un clúster más liberal mercantilizador a la "americana" y otro de orientación continental bismarckiana (Potucek, 2006). 
- Anglosajón. Inicialmente de vocación universalista (Beveridge Report) se basa en transferencias de prestaciones públicas homogéneas. Suele accederse a sus servicios y subsidios, de un carácter residual, tras la demostración de insuficiencia de recursos económicos de los beneficiarios (means testing). Se asume que los ciudadanos comprarán en el mercado buena parte de sus servicios de bienestar. En los últimos años se ha intensificado un giro hacia una mayor mercantilización individual en la procura del bienestar social, en paralelo a una creciente flexibilidad y desregulación laborales.

- Nórdico. Se basa en la combinación de ideas de solidaridad con el crecimiento económico y el pleno empleo, y la minimización de la dependencia familiar. Financiado mediante los impuestos generales, se caracteriza por un alto grado en provisión universal de servicios sociales asistenciales y personales, así como por unas prestaciones económicas generosas. La participación del mercado y la familia en la producción de bienestar es comparativamente menor. La igualdad de oportunidades y la homogeneidad de los grupos sociales en una ensanchada clase media legitiman la alta intervención pública.

- Mediterráneo. Conformado por unos estilos de vida en donde la familia se constituye como factor esencial de microsolidaridad y bienestar colectivo. La acción de la familia complementa sustancialmente la provisión estatal y de servicios ofertados por las organizaciones privadas con y sin ánimo de lucro. Existe una clara demarcación entre el sector contributivo, mediante prestaciones y servicios de mejor nivel otorgados a trabajadores "formales", y el no contributivo respecto a trabajadores "informales" y ciudadanos "precarios" y "sumergidos".

La diversidad de encajes institucionales y "lógicas del bienestar" de los regímenes sociales europeos no debería minusvalorar el proyecto común a todos ellos de una mayor solidaridad institucionalizada. No parece una mera contingencia histórica que el Estado del Bienestar haya sido "inventado" en el "Viejo Continente" y haya alcanzado una alta maduración institucional y legimitimación social. Existe una discusión abierta respecto a si Europa seguirá la opción liberal anglosajona, representada principalmente por EE.UU., respecto a una mayor individualización y mercantilización. El debate sobre quién merece ayuda y solidaridad (deservingness) ha crecido en intensidad en relación con algunas consecuencias asociadas al fenómeno de la inmigración y a la dualidad "ellos y nosotros" (Van Oorschot, 2006). Se denota, a este respecto, un cierto cambio en las actitudes ciudadanas y un énfasis en que los ciudadanos sean también responsables de su activación y del cuidado de sí mismos.

¿Hasta qué punto los planteamientos normativos y sociales planteados en el modelo social europeo podrían permitir contrarrestar o apoyar las tendencias ideológicas hacia una individualización de los riesgos sociales? Dado este marco de diversidad normativa e institucional, ¿cómo se plasmaría el proyecto de un modelo social europeo? Ante tal situación, se ha articulado el Método Abierto de Coordinación, como 16 principal instrumento de gobernanza europea. 


\section{EL MÉTODO ABIERTO DE COORDINACIÓN Y LA GOBERNANZA EUROPEA}

¿Cómo superar el dilema anteriormente planteado entre la necesidad, por un lado, de reforzar la regulación supranacional de la cuestión social, pero por otro lado, la conciencia de la gran diversidad en el interior de Europa? Una regulación basada, por ejemplo, en directivas europeas cuyos criterios vayan más allá de los modestos niveles aceptados por todos los estados miembros sería particularmente polémico. Una alternativa a esta regulación es el así llamado Método Abierto de Coordinación (MAC), que ha sido definido, por parte de algunos autores, como un estado post-regulatorio, y por otros, como un nuevo paradigma de regulación social?.

Esta regulación es “abierta”, según una regulación "blanda” (soft regulation), a fin de ajustarse a las nuevas condiciones económicas, a la enorme variación de situaciones en el interior de Europa, y al principio de subsidiaridad que caracteriza a este proyecto europeo. En este sentido, esta regulación no se basa en reglas detalladas, sino más bien en la instauración de "procedimientos" generales (regulación procedimental) que permiten una mayor flexibilidad, variación y libertad.

El calificativo de "abierto" en el MAC alude fundamentalmente a tres significados: (1) el concepto "abierto" refiere al carácter no obligatorio en el que se basa esta regulación. Si bien se trata de promover objetivos e indicadores comunes, la forma de llevarlos a cabo es abierta, en función de elecciones políticas y situaciones sociales a nivel nacional; (2) se entiende "abierto" como opuesto a burocrático, esto es, abierto a la discusión, pudiéndose así ajustar a posibles cambios en la situación económica y social o a los resultados de evaluaciones de políticas previas; y (3) es "abierto" ya que promociona la participación de diversos actores sociales. Trata de estimular la cooperación y participación de diversos actores que pertenecen a varios niveles -geográfico, social, administrativo, etc.- bajo el principio de "partenariado".

\section{Principios que gobiernan el Método Abierto de Coordinación}

El complejo estatus político ${ }^{8}$ de las instituciones europeas explica las recientes mutaciones en sus procedimientos de gobernanza del empleo y de la exclusión so-

$7 \quad$ Este método consiste en las siguientes etapas:

- Las instituciones europeas proponen una serie de líneas directrices, que consisten en medidas y objetivos generales, muchas veces, agrupados en torno a conceptos ("activación”, "empleabilidad", etc.).

- Estas líneas directrices son traducidas en políticas nacionales y regionales por parte de los estados miembros (Planes de Reforma Nacional).

- Se identifican una serie de indicadores (benchmarking) con los que comparar sincrónicamente (entre países) y diacrónicamente (a lo largo del tiempo) las evoluciones de los EE.MM. e identificar lo que han sido denominadas "mejores prácticas” (best practices).

- Se genera un proceso de evaluación, revisión y control por parte del grupo de pares y de las instituciones europeas.

8 Las instituciones europeas se ven abocadas a negociar la legitimidad de sus acciones respetando, a un mismo tiempo, la gran diversidad de filosofías políticas y mercados de trabajo en los estados 
cial. De una regulación exclusivamente basada en actuaciones legales (directivas europeas, por ejemplo) o institucional de carácter económico (como es el caso con la Unión Económica y Monetaria, UEM), se ha pasado a una coordinación que apela a la voluntad para cooperar por parte de los EE.MM. (legislación blanda o soft law). Los modos de regulación de la cuestión social en manos de las instituciones europeas se han diversificado. Así, al menor uso de directivas se han incorporado modos complementarios de regulación, como son el MAC, la presión del grupo de pares ${ }^{9}$, el fomento del diálogo social a nivel europeo y la política redistributiva de los fondos estructurales.

La extensión de estos modos de gobernanza se ha realizado en un contexto de emergencia de nuevos principios de regulación social, cuya característica común es la oposición a modos de regulación más centralizados y burocráticos, por considerarse excesivamente rígidos o inadaptados a las condiciones de diversidad normativa y económica que caracterizan a los países miembros de la Unión Europea. Se trata de la movilización y multiplicación de recursos de coordinación alternativos dirigidos a promover la autorregulación a nivel descentralizado como son los procedimientos de benchmarking, el intercambio de buenas prácticas (best practices) o la promoción del partenariado (Walters y Haahr, 2005).

EI MAC propone una regulación dirigida a la convergencia en términos de resultados, una vez acordados conjuntamente principios generales e indicadores comunes, pero dejando a los estados miembros (EE.MM.) la posibilidad ("abierta”) de optar por los instrumentos adecuados para llevar a cabo estos objetivos, y de ajustarlos a la diversidad de elecciones políticas y coyunturas sociales a nivel nacional. Solo la definición de objetivos se produce a nivel central, pero la implementación se realiza de forma descentralizada. Estos objetivos, propuestos por las instituciones europeas, consisten en principios generales, en vez de propuestas específicas. Tales propuestas generales son traducidas por parte de los EE.MM. en políticas nacionales, y en algunos casos en políticas regionales.

El MAC consiste así en un nuevo modo de gobernanza articulado en torno a tres ejes: (A) frente a la formalización sustantiva de la norma, la desformalización y desustancialización de los recursos normativos (regulación procedimental); (B) frente a la centralización normativa, la descentralización deliberativa (gobernanza inter-normativa a múltiples niveles); y (C) frente a la regulación exógena, la movilización de recursos alternativos con los que promover la autorregulación.

Respecto al primer aspecto (A), el MAC implica un acuerdo entre los EE.MM., no tanto respecto al contenido de los preceptos sociales, sino más bien de sus rituales

miembros. Esta legitimidad se articula en torno a tres ejes, legitimidad técnica (basada en la búsqueda de eficacia), legitimidad política (profundización democrática) y legitimación social (ofrecer oportunidades políticas para los diversos miembros de la sociedad civil).

9 Los resultados de las políticas de empleo nacionales son sometidos a un examen público por parte de los otros EE.MM., facilitándose la comparación explícita con los resultados de diversos países, y

18 adoptando como base una serie de indicadores previamente acordados. 
y procedimientos, de los mecanismos y modalidades de negociación de estos preceptos, de tal modo que estos son sometidos a un proceso de progresiva reinvención. Se promueve una regulación procedimental (difusión de rutinas procedimentales), que consiste en el establecimiento de una serie de "rituales"10 dirigidos a la difusión de paradigmas epistémicos estructurados en torno a conceptos que se difunden a nivel nacional -tal y como veremos más adelante- con el ejemplo específico de la activación.

La difusión de estos conceptos contribuye a enmarcar los debates de los estados miembro acerca de las cuestiones de empleo y cohesión social, ejerciendo así una importante influencia socio-cognitiva"1. Este poder regulador de la Unión Europea, consistente en su capacidad de armonización de ideas, representaciones y metas políticas (Palier, 2001), explica el importante papel que juega el marco interpretativo europeo en la rearticulación de la cuestión social a nivel nacional. Por tanto, la naturaleza de la regulación europea en el dominio social y del empleo se basa en su capacidad de difusión de paradigmas de concepción y articulación de la cuestión social. La peculiar fuerza reguladora de las instituciones europeas radica en su capacidad de intervenir en una política de producción de sujetos. Así, “... el poder funciona de acuerdo a los modos y maneras mediante los cuales nos gobernamos a nosotros mismos, estimulándonos a adoptar tales subjetividades como son el buscador de empleo activo, los ciudadanos empoderados o el consumidor discerniente..." (Walters y Haast, 2005: 13).

Un segundo rasgo (B) que caracteriza al MAC es su carácter internormativo (gobernanza a múltiples niveles). La promoción de este modo de gobernanza permite responder a la crisis de legitimidad democrática ante la que se enfrentan las instituciones europeas. Sustituye una perspectiva centralizada, basada en un modelo formal de subordinación, por un modo de regulación que busca la implicación de gran número de actores en los procesos de toma de decisiones y ajustar así una gama tan amplia de perspectivas políticas e intereses sociales. Con este modelo de gobernanza a diversos niveles, las instituciones europeas negocian legitimidad no solo a nivel político (respondiendo, así, al profundo déficit democrático del que se acusa a las instituciones europeas), sino también a nivel técnico (rendimiento estadístico y eficacia social).

Objetivo nuclear, por tanto, es la promoción de un estado reflexivo dirigido a incentivar diálogos entre políticas y la creación de espacios de deliberación mutua y negociación constante entre diversos grupos y actores sociales. Se trata de estimular la cooperación y participación de diversos niveles -geográfico, social o administrativo- bajo el principio de "partenariado" (gobernanza a múltiples niveles verticales y horizontales). La movilización de diversos niveles y actores persigue la consecución consensuada y

11 Para una discusión de los cambios sintácticos y semánticos integrados en el paradigma de la activación, véase Serrano Pascual (2004). 
negociada de los fines acordados. Se pretende impulsar a las políticas sociales hacia un estado reflexivo y fomentar de este modo un cierto aprendizaje político.

Esta apelación a la participación plural de gran número de actores sociales y políticos para el establecimiento de sus propuestas permite ajustarlas, de forma abierta, a posibles cambios en la situación económica y social o a los resultados de evaluaciones de políticas previas. Este modelo de regulación podría así calificarse como de "invención deliberativa" de un orden normativo, en función de registros múltiples, que explica el carácter contingente y continuamente revisado de sus propuestas.

Estos procesos de internormatividad deliberativa promueven una negociación reflexiva de los procedimientos y los métodos, así como del contenido de las propuestas. Las propuestas pueden entenderse de este modo como compromisos políticos entre voces asimétricas, lo que explica la creatividad y fertilidad terminológica de las instituciones europeas, así como el carácter híbrido de sus conceptos (como es el caso de la noción de flexiseguridad, por ejemplo).

Dado el importante poder simbólico en manos de las instituciones europeas, diversos actores luchan por espacio político en el seno del discurso europeo. La arena europea puede así entenderse como un espacio para las luchas políticas, que no serían sino batallas cognitivas por el poder para imponer una visión del mundo social, así como de las representaciones sociales y categorías bajo las que se perciben los problemas nacionales. Los conflictos políticos se articulan, aun parcialmente, como conflictos semánticos. El espacio supranacional se convierte en un ámbito para la lucha de ideas y enfrentamiento político de diversas voces.

Una tercera característica (C) de este método de coordinación es la de dirigirse a la promoción de la autorregulación. Este modo de gobernanza puede entenderse como inspirado en las nuevas éticas de gestión empresarial (Alonso, 2007). Son incorporadas metáforas procedentes del mundo económico, en particular, de los nuevos modos de gestión organizacional dirigidos a la regulación actitudinal, tales como la noción de benchmarking o best practices. Se trata de inducir en los EE.MM. una lógica competitiva de rendimiento estadístico (Salais, 2006) y de otorgar espacios de autonomía con el objetivo de mejorar la eficiencia.

El objetivo es estimular una lógica de optimización del "rendimiento social" en el interior de la UE. Esta colonización de metáforas procedentes del mundo empresarial se ve plasmada en su particular modo de gestión por objetivos. Se establecen una serie de indicadores que cuantifican los objetivos con el fin de medir cuantitativamente las evoluciones de los diversos EE.MM. hacia la consecución de estos y se define un plazo en el que estos objetivos tienen que ser realizados ${ }^{12}$. Se contribuye a las prácti-

12 Se han definido una serie de indicadores cuantitativos con los que medir y regular la evolución de los mercados de trabajo de los estados miembros, como son, una tasa de empleo del $67 \%$ en el 2005 y del $70 \%$ en el 2010; una tasa de empleo para las mujeres del $57 \%$ en el 2005 y del $60 \%$ en el 2010 ; y una tasa 
cas de visibilización de los "rendimientos sociales” a través de gráficos y cuadros comparativos que estimulan una estrategia de autogobierno. Las instituciones europeas promueven así una lógica competitiva entre los EE.MM., sometiéndoles a un continuo proceso competitivo de autosuperación y estableciendo un método de continua evaluación de progreso y de sistemática comparación ${ }^{13}$, diacrónica -entre países-, y sincrónica -a lo largo del tiempo- a fin de controlar la evolución de los EE.MM. Se trata de fomentar que las instituciones europeas, en sentido amplio, se transformen en organizaciones que "aprenden” (Walters y Haahr, 2005).

Por tanto, dado el peculiar modo de gobernanza europea, tres son los principales instrumentos potenciales de este método: su capacidad persuasora y socializadora, la posibilidad de otorgar oportunidades políticas y recursos estratégicos para los diversos miembros de la sociedad civil, y finalmente la capacidad de proporcionar instrumentos metodológicos para estimular la eficacia de estas políticas (útiles estadísticos, nuevas instituciones, etc. $)^{14}$. Un ejemplo emblemático del modo de funcionamiento del MAC es la difusión del paradigma de la activación. El metaanálisis de este principio nos permitirá sacar algunas conclusiones acerca de las potencialidades y limitaciones de este modo de regulación.

\section{EL NUEVO PARADIGMA ACTIVADOR Y SU AMBIVALENTE EVALUACIÓN}

El peculiar estatus político del MAC de las instituciones europeas, y el carácter internormativo que define las propuestas europeas, así como el importante poder simbólico que ejercen dichas instituciones, explica la naturaleza polisémica y ambigua de gran parte de los conceptos que se articulan. El carácter híbrido de estas propuestas explica la naturaleza paradójica que adoptan, tal y como veremos en el caso del discurso europeo sobre la activación.

Esta doble naturaleza, polisémica y paradójica, puede estudiarse como reveladora del carácter polifónico de las propuestas europeas, al ser resultado de la pluralidad de voces autónomas que habitan tras sus conceptos, de tal modo que filosofías políticas y acentos dispares pueden ser transversales a sus propuestas.

\footnotetext{
de empleo del 50 \% para trabajadores mayores ( 55 a 64 años) en el 2010; cada desempleado debe poder acceder a una ayuda antes de llegar a seis meses de desempleo en el caso de los jóvenes y a 12 meses en el caso de los adultos, bajo la forma de formación, re-educación, prácticas laborales, una oferta de trabajo o una medida de empleabilidad, combinado, cuando sea necesario, con asistencia para la búsqueda de trabajo; en el año 2010, el 25 \% de los desempleados de larga duración participará en una medida activa, bajo la forma de formación, re-educación u otro tipo de medida dirigida a la empleabilidad.

13 La estrategia europea incita a los estados miembros a comparar sus propios resultados con los de otros países, pudiéndose así impulsar a los actores a redefinir colectivamente los objetivos y las políticas. El objetivo es organizar un proceso de difusión de conocimientos y representaciones sociales a nivel europeo. Este ejercicio permite la comparación de los resultados de cada país con los de su grupo de pares, y el uso de indicadores pretende incitar a la mejora continua de estas políticas.

14 Para una reflexión acerca de cómo se traducen estas potencialidades en la práctica en el caso de la promoción de la igualdad de oportunidades y de una perspectiva integral de género, véase Serrano Pascual (2008).
} 
Esta interacción de voces polifónicas explica el carácter mixto de gran parte de dichos conceptos. No se trata solo de la mezcla de voces plurales, sino también de la coexistencia de puntos de vista que se enfrentan entre sí. Por ejemplo, el mezclar, como lo hace el concepto de flexiseguridad, la noción de flexibilidad y la de seguridad, que evocan dos marcos de regulación del trabajo dicotómicos, no hace sino mostrar la pervivencia, en una misma noción, de punto y contrapunto ${ }^{15}$. Semejantes palabras híbridas realizan un importante trabajo semántico. Donde había solo un pensamiento, las instituciones producen un desdoblamiento y un deslizamiento "bajtiano" de sentido. Apropiándose de una noción, como es la de seguridad, ajena y contrapuesta al discurso de la flexibilidad, e integrándola en su propio discurso, la vuelve bi-vocal y confiere así otra orientación semántica a la noción de flexibilidad.

De este modo, las nociones nucleares del discurso europeo responden a diversos puntos de vista, y son reflejo del particular modo de regulación, al que nos hemos referido en líneas anteriores, basado en la gobernanza a múltiples niveles. Un ejemplo ilustrativo de este modo de regulación es la apelación al mundo científico, y a los así llamados "expertos", para la conformación deliberativa (y justificación) de las propuestas sociales de las instituciones europeas. Esta apelación al mundo científico permite que estas propuestas aparezcan como políticamente neutras.

Dada la complejidad de las filosofías políticas en el interior de la UE, y la diversidad de intereses que predominan entre los actores que participan en este proyecto, las instituciones europeas están sometidas a una constante apelación a la neutralidad. Buena parte de la legitimidad europea se basa en el establecimiento de espacios deliberativos que hacen del espacio europeo un foro donde se expresa la pugna de ideas entre diversos actores sociales (voices). Se colige, por tanto, que las instituciones europeas deban aparecer como receptoras neutrales de las ideas resultantes del diálogo y deliberación entre tales actores y los niveles múltiples de interacción. Así, la complejidad y diversidad de centros de poder en el interior de las instituciones europeas, ha favorecido que diversos lobbies y think-tanks hayan sido incorporados como parte de la burocracia europea. Términos, como "expertocracia" o "femocracia", usados en el análisis de estos procesos de regulación supranacional, denotan la contribución de esta pluralidad de grupos de profesionales en la producción de "discursos" en la UE. Esta pluralidad de poderes en el seno del proyecto europeo explica la importancia de entender la gobernanza supranacional como “... una actividad problematizadora [ya que] las luchas políticas son también conflictos sobre los significados..." (Walters y Haahr, 2005: 6).

La participación asimétrica de voces plurales explica la peculiar naturaleza de gran parte de los conceptos europeizadores. En el seno de sus conceptos se integran nociones paradójicas [flexi-seguridad; activ-a(c)ción, emple-(h)abilidad], que auspiciando marcos que apelan a la emancipación individual, plantean, sin embargo, políti-

15 Al igual que señala Bajtin (1979/2004), cuando destaca cómo los héroes de Dostoyevski conversan con sus caricaturas (Iván Karamázov con el diablo, por ejemplo). 
cas que pueden resultar, en algunos contextos, profundamente regresivas. Tal es el caso con el concepto de activación.

\section{El significado de la activación y sus rasgos}

Por activación entendemos un tipo de intervención social de los poderes públicos dirigida a la movilización y a la mejora de la adaptabilidad de la mano de obra en general y de los desempleados, en particular. El paradigma de intervención basado en la activación, se caracteriza fundamentalmente por tres rasgos: su perspectiva individualizadora, el énfasis en el empleo y la importancia del principio de la contractualización ${ }^{16}$. Este paradigma de intervención plantea un proceso de creciente individualización en el tratamiento del problema de exclusión en el mercado de trabajo, en un doble sentido (1) se hace al sujeto individual el eje nuclear de las intervenciones (y por tanto, parte del problema) y, (2) se extiende la tendencia, propia de este tipo de paradigma, a fomentar la participación del individuo en su proceso de integración ${ }^{17}$. Ambos aspectos confluyen, sin embargo, en el énfasis creciente en reclamar a los "clientes" o "usuarios" que actúen como ciudadanos responsables.

Este paradigma induce una transformación en la atribución de responsabilidades frente a la cuestión social y replantea el ámbito de las cuestiones susceptibles de ser problematizadas. Frente a un Estado garantizador de derechos sociales (entitlement state) se reclama un Estado dirigido a facilitar la adaptabilidad de los individuos (enabling state), y cuya función sería fundamentalmente la de responsabilizar a los ciudadanos y facilitarles instrumentos para incrementar su empleabilidad. La referencia a la solidaridad (responsabilidad colectiva), como legitimadora de la acción pública, está siendo desplazada por un énfasis creciente en la responsabilidad del individuo. Esta creciente apelación a la responsabilidad individual otorga legitimación al carácter coactivo que adquieren en numerosas ocasiones estos programas de activación. Su base justificativa se apoya en el principio moral (y por tanto universal) que se deriva de los deberes que cada individuo (ciudadano) contrae con el Estado y con la colectividad.

Se parte de una concepción psicologicista de las relaciones sociales, según la cual, la activación consistiría en un proyecto individualizador dirigido a la autonomización de los sujetos (Franssen, 2003). En este discurso acerca de la dependencia y de la responsabilización, el lenguaje de los “derechos", articulado en torno a la provisión de seguridad social como responsabilidad colectiva, está siendo progresivamente sustituido por un discurso que apela a la ética de la responsabilidad (Dean, 2003). El (2007).

16 Para una discusión detallada acerca del paradigma de la activación, véase Serrano y Magnusson

17 En el caso español, esta tendencia se ha visto reforzada tras la Ley de Empleo del 2003, en donde se acentúa la condicionalidad del seguro de desempleo incidiendo en la obligación, por parte de los demandantes de empleo, de participar en medidas activas, a partir de la firma de un compromiso de actividad con el que el desempleado acredita su disponibilidad. 
individuo aparece crecientemente como el único culpable de las decisiones equivocadas que pueda adoptar (Bauman, 2001).

Este tipo de prácticas contienen un doble componente complementario de presiones exógenas y endógenas. Por un lado, se trata de influir, a través de sanciones (por ejemplo, limitando el acceso a la protección social), en los comportamientos de los sujetos, pero, por otro, se trata de prácticas biopolíticas dirigidas a la producción de sujetos "normalizados". Esta producción de sujetos se lleva a cabo a partir de tres prácticas consistentes en (a) el disciplinamiento/normalización, (b) la vigilancia y (c) la intervención terapéutica.

El sujeto objeto de intervención es considerado como susceptible de disponer de una serie de déficits, de formación, en unos casos, y/o de voluntad o personalidad, en otros. Así se está extendiendo en gran número de países europeos un modelo de intervención paternalista cuyo rasgo fundamental sería la medicalización terapéutica (Schram, 2000). La dependencia pasa a convertirse en una patología.

Problemas económicos y políticos se transforman en cuestiones de motivos y voluntades personales, estimulándose la despolitización de la gestión del conflicto social. Se omite la emergencia del carácter sociopolítico de la exclusión social, al anular el nexo causal que pueda establecerse con la relaciones de poder y dominación ${ }^{18}$.

\section{La naturaleza paradójica de la activación}

Esta noción, al igual que otras que articulan el discurso de las instituciones europeas, presenta un importante carácter paradójico. Este carácter paradójico es el resultado de su ubicación semántica en un espacio de inter-textualidad, tal y como se desarrolló en un momento anterior. Como resultado de este proceso intertextual el discurso de la activación se adapta tanto a registros social-demócratas como de corte neoliberal. Así, este discurso mantiene una posición híbrida entre, por un lado, la apelación a marcos de empoderamiento (empowerment) de los individuos frente a las instituciones y, de otro, defienden modelos de intervención que no posibilitan en muchos casos mayor poder de intervención que la adaptación a las leyes del mercado. Activar sería así propiciar la adaptabilidad personal, la disponibilidad del sujeto ${ }^{19}$.

En primer lugar, la noción de activación evoca explícitamente un concepto ontológico en el cual el sujeto sería moralmente autónomo y autodeterminado, independiente y responsable, gobernado por la libre voluntad, esto es, un sujeto auto-

18 Para un interesante análisis acerca de la emergencia de categorías sociales que polarizaron el debate acerca de la exclusión “involuntaria” del trabajo, y con estas, el carácter social y político que adquiere el problema, véanse las reflexiones de Salais et al. (1986) y Topalov (1994).

19 Para una discusión más detallada acerca de las paradojas de la activación, véase Crespo Suárez y Serrano Pascual (2007). 
gobernado $^{20}$. Sin embargo, por otra parte, se plantea una desconfianza implícita en los verdaderos motivos que guían al sujeto y le hacen vulnerables a las trampas del bienestar. De consecuencia, se pone en cuestión la noción de sujeto como moralmente autónomo. Esta concepción requiere así de sujetos "activados": individuos económicamente motivados por sanciones positivas o negativas. Se trata de una adaptación "pasiva" que implica más una capacidad reactiva, y que entiende a los sujetos como motivados por factores externos. Este discurso de la autonomía, movilidad, y calidad del trabajo contrasta con los instrumentos coactivos que se han establecido para aumentar la tasa de actividad y para inducir a los sujetos a trabajar. La activación pasa a ser entendida como la supervisión de la conducta y como instrumento de disciplina social.

En segundo lugar, este discurso activa marcos políticos contradictorios. Por un lado la metáfora de la activación apela a la emancipación ciudadana como el principal objetivo. Así, este discurso es rico en metáforas vinculadas a la lucha y al combate (lucha contra el desempleo, combate de la pobreza, o batalla contra la exclusión, pongamos por caso), a fin de reforzar el dogma de dinamismo en el que se basa el discurso de la activación. Sin embargo, por otro lado, se designan estrategias dirigidas al cambio psicológico individual, el cambio en las motivaciones, en las actitudes y conducta. Las políticas se convierten así en procedimientos terapéuticos dirigidos a la motivación y al cambio psicológico. En este sentido, este discurso sobre la activación se caracteriza por naturalizar el cambio, los condicionantes económicos, usando gran número de abstracciones (Serrano y Crespo, 2007). El cambio hacia la sociedad basada en el conocimiento se presenta como una realidad ante la cual no queda más elección que aceptar. La única respuesta política sería tratar de beneficiarse lo más posible de esta situación. La aspiración a la autosuficiencia, intervención y autonomía contrasta con la apelación al pragmatismo, a la necesidad de adaptarse a una nueva y paradójica pasividad.

En tercer lugar, las implicaciones socio-políticas de este discurso entran también en contradicción. Por un lado, se defiende la activación económica, pero se fomenta, a un mismo tiempo, la pasividad política. El mercado es construido bajo el carácter de ley, promocionándose la desaparición de las condiciones políticas para ejercer la autonomía, como son los espacios desmercantilizados, y pudiendo facilitar la inseguridad y vulnerabilidad en el mercado (Alonso, 2007). Se hace uso del concepto de dependencia para descalificar formas previas de intervención (dependencia del Estado); sin embargo, este uso del concepto representa solo una forma de entender la dependencia. De hecho las mal Ilamadas políticas "pasivas" fueron producidas como espacios de desmercantilización y de emancipación de las condiciones de heteronomía y vulnerabilidad que caracterizaban las relaciones laborales regidas por las leyes del mercado "libre". Por tanto, las así llamadas políticas "activas" permiten combatir la

20 El sujeto requerido por este discurso es una persona analítica capaz de sopesar cuál es la mejor alternativa en un contexto creativo e interdependiente, con un alto grado de control del proyecto personal, y que actúa en función de sus propias convicciones y principios, de sus propias éticas. 
dependencia económica (de las instituciones, de la familia), pero promueven, a su vez, la dependencia política (del mercado).

El discurso acerca de la activación parece ir dirigido a la promoción de dinamismo económico, pero pudiendo provocar, a un mismo tiempo, resignación y pasividad política. En otras palabras, los sujetos disponen de un alto grado de autonomía para adaptarse a las reglas cambiantes del juego, pero esta autonomía no les permitirá poner en cuestión estas mismas reglas. Ciertamente este tipo de propuestas pueden ser capacitadoras y empoderadoras cuando la configuración institucional e ideológica de un país refuerza la posición del trabajador y, por tanto, una orientación semántica emancipadora. Pero cuando el equilibrio de fuerzas es desfavorable para el trabajador también pueden contribuir a la fragilización de este. Esta situación plantea una de las más importantes limitaciones de este proceso de europeización, al disponer de un importante poder de difusión y problematización de modos de nombrar la cuestión social, pero con un escaso poder a la hora de transformar la configuración institucional y el (des)equilibrio de fuerzas en un determinado país. Esta situación se ve acompañada de otras cuestiones que pueden contribuir a fragilizar el Modelo Social Europeo, tal y como se desarrolla en el próximo apartado.

\section{EL PROCESO DE LUXEMBURGO Y LA AGENDA DE LISBOA: OBJETIVOS EUROPEOS Y MEDIOS NACIONALES}

El Método Abierto de Coordinación y el cambio paradigmático hacia la activación laboral son hitos de la reciente europeización. Ambos encuentran un reflejo programático e institucional en el denominado Proceso de Luxemburgo y la Agenda de Lisboa (o estrategia de Lisboa). Ciertamente el intento de la Unión Europea por conciliar planteamientos generales y enfoques nacionales ha constituido la actuación más decidida por asegurar una participación del nivel comunitario en el campo de las políticas sociales y, en particular, en lo que atañe a la inclusión social. Como se ha indicado líneas arriba, las instituciones de la Unión Europea constituyen un ámbito crucial en la pugna por significantes y significados. Así, el ser capaz de influir en las ideas de los ámbitos nacionales ha configurado una estrategia comunitaria prioritaria de política y políticas relativas al bienestar social.

El proceso de Luxemburgo iniciado en 1997 y la Agenda de Lisboa de 2000 inauguraron una nueva forma de intervención comunitaria en lo que atañe a políticas de empleo, inclusión social y pensiones. El objetivo no era tanto el de armonizar instituciones y legislaciones nacionales, como el de compartir concepciones, conocimientos, ideas, normas de acción y visiones en pos de una convergencia en los programas nacionales. Se trataba, por tanto, de lograr gradualmente un enfoque político común respecto a la protección social en la Unión Europea. Empero, no se descartaron, tal y como se ha planteado, la consecución de metas concretas. Ya en 1999, justo antes de la introducción de la moneda única, la Comisión Europea publicó un documento en el 26 cual se renovaba el compromiso por parte de la UE de profundizar la cooperación 
existente a nivel comunitario. Fueron cuatro las áreas identificadas como cruciales para la articulación de una estrategia concertada: (1) promover la inclusión social; (2) rentabilizar el trabajo (make work pay) y proveer seguridad de rentas; (3) garantizar las pensiones y hacer sostenibles los sistemas pensionísticos; y (4) asegurar un nivel de calidad alto y la viabilidad financiera de la atención sanitaria (CEC, 1999).

Con relación al proceso de Luxemburgo, en el Consejo de Lisboa de marzo de 2000 todos los EE.MM. se comprometieron a incrementar las tasas nacionales totales de empleo (como ratios entre el total de la población empleada y la población laboral activa entre los 15 y los 64 años) a un mínimo del 70 por ciento en el año 2010 (60 por ciento en el caso de las mujeres). En la reunión del Consejo celebrada un año después de Estocolmo, se adoptó un acuerdo similar con el fin de alcanzar en 2010 un porcentaje al menos del 50 por ciento en las tasas de empleo de las personas entre 55 y 64 años $^{21}$.

Con el proceso de lucha contra la pobreza y la exclusión social iniciado en la cumbre de Lisboa, y tras el Consejo de Niza de diciembre de 2000, se completaron dos Informes Conjuntos sobre Inclusión Social (2002 y 2004) que incorporaron los resultados de los Planes Nacionales de Acción para la Inclusión Social. Con posterioridad se han elaborado los Informes Conjuntos anuales durante el periodo 2005-2008 (CEC, varias fechas).

En líneas generales los Informes Conjuntos han perseguido incrementar la eficacia de las políticas sociales de los EE.MM. en la promoción de la inclusión social incentivando la colaboración entre decisores públicos (policy-makers), agentes sociales, ONG, académicos y los propios excluidos. Tras varios años de puesta en práctica del proceso de Luxemburgo y la Agenda de Lisboa se han producido evaluaciones críticas de variada índole.

Reproduciendo, en alguna manera, la doble visión articulada por las Direcciones Generales II (Economía y Asuntos Financieros) y V (Empleo y Asuntos Sociales), la evaluación política del proceso de Luxemburgo y la Agenda de Lisboa descansa en buena medida en los enfoques macroeconómicos divergentes de ambas Direcciones Generales. De una parte se ha hecho hincapié en la visión de las políticas económicas ofertistas (suply-side) en la línea de lo expresado desde el "Consenso de Washington" por otros organismos internacionales, tales como el Banco Mundial o la OCDE (Trubeck y Mosher, 2003). Así, se asevera que la "Estrategia de Lisboa" habría fallado institucionalmente porque un método de gobernanza basado en la "moralidad" ("gobierno de las voluntades") no es ni suficiente ni eficiente. En la misma línea de argumentación se señalaba que los EE.MM. no deberían ser recompensados como "virtuosos" o castigados como "viciosos" de acuerdo a sus rendimientos (performance) (Creel et al., 2005).

21 La tasa de empleo no solo persigue alargar las vidas laborales de los trabajadores por cuenta ajena sino posponer la edad "efectiva” de jubilación. En el Consejo de Barcelona de marzo de 2002, por ejemplo, Italia se comprometió a elevar en cinco años la jubilación "real” para el año 2010. 
Alternativamente se ha subrayado el carácter soft (blando) y friendly (amable) que necesariamente deben tener las iniciativas políticas e institucionales en esta fase del largo proceso de europeización. Además, se ha destacado que el proceso de Luxemburgo y la agenda o estrategia de Lisboa han favorecido una movilización de actores implicados y organismos de la gobernanza múltiple europea que han intercambiado información (Moreno et al., 2003; Ferrera, 2005). En algunos casos se ha estimulado -aun indirectamente- el "efecto emulación" o "alcance" (catching-up) como el espectacular incremento en las tasas de participación laboral femenina en el caso de España22.

\section{COMENTARIOS FINALES}

Las estrategias promovidas por las instituciones europeas para regular el empleo y la cohesión social presentan un indudable potencial en términos de eficacia política y de cohesión social. En primer lugar, las instituciones europeas han estimulado ciertas representaciones acerca de la cuestión social y han extendido un cierto vocabulario en los discursos políticos nacionales (empleabilidad, partenariado, activación, perspectiva integral de género, pongamos por caso), posibilitando, en algunos casos, la sensibilización política y pública frente a la situación de ciertos grupos en el mercado de trabajo (mujeres, trabajadores mayores, excluidos sociales). Ello ha permitido que tales expresiones aparezcan definidas bajo el estatus de "problemas", lo que supone una condición necesaria y preliminar para la movilización política. En segundo lugar, parece haber facilitado la provisión de herramientas políticas para algunos grupos de la sociedad civil (por ejemplo, movimientos de mujeres o agentes sociales en algunos países). Finalmente, esta estrategia ha promovido una cierta concienciación acerca de la necesidad de disponer de herramientas metodológicas adecuadas (mejores indicadores estadísticos, desarrollo de estructuras e instituciones para el seguimiento de estas políticas, y la evaluación de las políticas de empleo). Estos tres ejes, que representan el potencial del MAC de las políticas de empleo, también otorgan a las instituciones europeas legitimidad técnica (basada en la búsqueda de eficacia), política (profundización democrática) y legitimación social (sensibilidad pública).

Sin embargo, hay también importantes áreas en las cuáles la estrategia presenta importantes debilidades: por ejemplo, los rituales de cumplimiento de las propuestas europeas pueden transformarse en un mero ejercicio formal, su carácter excesivamente "abierto" -demasiado dependiente de la actitud nacional-, inestable, asimétrico, y potencialmente abierto a la desregulación. El escaso margen de maniobra otorgado a terceros actores, la escasa capacidad para llevarse a cabo de una forma

22 En el periodo 1995-2006, la tasa de empleo femenina española ha sido la de mayor crecimiento en la UE-15 (22,5 puntos desde el 30,7\% a un 53,2\%). Para una comparación de las dinámicas del crecimiento del empleo formal femenino entre las países de "vanguardia" nórdicos y los "retrasados" de la Europa del 
transversal, con la cooperación de diversos departamentos administrativos, o la dificultad de transferir "buenas prácticas" constituyen también grandes retos por resolver. Entre todos estos, nos hemos concentrado principalmente en cuatro. En primer lugar, el MAC promociona objetivos generales basados en nociones paradójicas. Al no haber apenas margen para la intervención en el marco institucional, se refuerzan las relaciones de fuerza (asimétricas) existentes en el propio país. Los conceptos propuestos y su carácter polifónico, más que reforzar el diálogo entre políticas, legitiman con frecuencia las voces y representaciones hegemónicas.

En segundo lugar, se mantiene una orientación casi exclusivamente dirigida a la intervención sobre la oferta de mano de obra, contribuyendo a la difusión de un marco individualizante de representación de la cuestión social. Si en cuestiones como la empleabilidad y la activación, así como en la igualdad de oportunidades, la estrategia europea parece haber jugado un papel central, al menos en la orientación de debates públicos y discusiones políticas, su impacto ha sido muy limitado en otros aspectos como cambios organizacionales, políticas impositivas, calidad del trabajo y creación de empleo.

En tercer lugar, parece necesario reforzar la participación de otros agentes sociales y favorecer la cooperación entre los diversos actores implicados, ya que el proceso adolece de ser excesivamente jerárquico y burocrático, contribuyendo así a alimentar las acusaciones realizadas al "déficit democrático" del proceso europeizador.

Finalmente, predomina un énfasis excesivo en criterios de cantidad, en detrimento de criterios como la calidad del empleo y de las condiciones de vida. Debe reiterarse que, con carácter general, el Modelo Social Europeo atañe a ambas dimensiones relativas al crecimiento económico y a la cohesión social. En base a ello, la legitimidad de los procesos de cambio de las políticas sociales en la Unión Europea es dependiente del apoyo a un sistema axiológico que valora lo social en igual medida, cuando menos, que lo económico. O que, sencillamente, no los desagrega.

\section{REFERENCIAS BIBLIOGRÁFICAS}

AER (2005), "The European Social Model must be grounded in diversity" <http:// www.a-e-r.org/en/news/2005/2005101301.html> (consulta: 26 mayo 2008).

Adnett, N. y Hardy, S. (2005), The European Social Model. Modernisation or Evolution? Cheltenham: Edward Elgar.

Alber, J. (2006), "The European Social Model and the United States", European Union Politics, 7(3): 393-419.

Alonso, L. E. (2007), La crisis de la ciudadanía laboral. Barcelona: Anthropos.

Bajtin, M. (1979/2004), Problemas de la poética de Dostoievski. México D.F.: Fondo de Cultura Económica.

Bauman, Z. (2001), En busca de la política. Buenos Aires: Fondo de Cultura Económica.

CEC (Commission of the European Communities) (1999), Commission Communication: A concerted strategy for modernising social protection. COM(99) 347 final. 
- (varias fechas), Joint Reports on Social protection and Social exclusion <http:// ec.europa.eu/employment_social/spsi/joint_reports_en.htm\#2008\#2008> (consulta: 29 mayo 2008)

Cowles, M., Caporaso, J. y T. Risse eds. (2001), Transforming Europe: Europeanization and Domestic Change. Ithaca, Nueva York: Cornell University Press.

Creel, J., Laurent, E. y Le Cacheux, J. (2005), “Delegation in inconsistency: The 'Lisbon strategy' Record as an Institucional Failure", Documents de Travail de l'OFCE (Observatoire Français des Conjonctures Economiques), Núm. 2005-07 <http://www.ofce.sciences-po.fr/pdf/dtravail/WP2005-07.pdf> (consulta 29 mayo 2008).

Crespo Suárez, E. y Serrano Pascual, A. (2007), "The paradoxes of the active subject in the discourse of the EU institutions", en R. van Berkel and B. Valkenburg, eds. Making it personal. Individualising activation services in the EU. Bristol: Policy Press.

Dean, H. (2003), “Human rights and welfare rights Re-conceptualising dependency and responsibility". Ponencia presentada en la "First Conference of the European Social Policy Research Network, Social Values, Social Policies", Tilburg, 2931 agosto, 2003.

Esping-Andersen, G. (1990), Three Worlds of Welfare Capitalism. Cambridge: Polity Press.

- (1993), “La macrosociología comparativa de los Estados del Bienestar”, en L. Moreno (ed.), Intercambio Social y Desarrollo del Bienestar. Madrid: CSIC.

- (1999), Social Foundations of Postindustrial Economies. Oxford: Oxford University Press.

ETUC (2005), "What is the "European Social Model” or "Social Europe"?" <http:// www.etuc.org/a/111> (consulta: 26 mayo 2008).

EuroActiv (2005), "Hans Skov Christensen, director general of the Federation of Danish Industry" <http://www.euractiv.com/en/socialeurope/hans-skov-christensendirector-general-federation-danish-industry/article-146400> (consulta: 26 mayo 2008).

Flora, P. (1993), “Los estados nacionales del bienestar y la integración europea”, en L. Moreno (ed.), Intercambio social y desarrollo del bienestar. Madrid: CSIC.

Ferrera, M. (2005), The Boundaries of Welfare. European Integration and the New Spatial Politics of Social Protection. Oxford: Oxford University Press.

Franssen, A. (2003), "Le sujet au coeur de la nouvelle question sociale", La revue nouvelle, 17 (12): 10-50.

Giddens, A. (2006), "Debating the Social Model. Thoughts and Suggestions", en The Hampton Court Agenda: a Social Model for Europe. Londres: Policy Network.

Guillén, A.M. y Palier, B. eds. (2004), EU Enlargement, Europeanization and Social Policy, Journal of European Social Policy, 10 (2) (monográfico).

Jepsen, M. y Serrano Pascual, A. (2005), "The European Social Model: an exercise in deconstruction”, Journal of European Social Policy 15(3): 231-245.

Lakoff, G. (2007), No pienses en un elefante. Madrid: Editorial Complutense.

30 Journal of Political Research, 42(2): 185-199. 
- (2007), “Europa social, bienestar en España y la 'malla de seguridad' en A. Espina (coord.), Estado de Bienestar y competitividad. La experiencia Europea. Madrid: Fundación Carolina-Siglo XXI.

- (2008), "The Nordic Path of Spain's Mediterranean Welfare”, Center for European Studies Working Paper Series, Harvard University, <http://www.ces.fas.harvard. edu/publications/docs/pdfs/CES_163.pdf> (consulta: 30 noviembre 2008).

Moreno, L., Matsaganis, M., Ferrera, M. y Capucha, L. (2003), “¿Existe una 'malla de seguridad' en la Europa del Sur? La lucha contra la pobreza y la exclusión en España, Grecia, Italia y Portugal”, Revista Internacional de Sociología, 36: 7-31.

Moreno, L. y McEwen, N. (2005), "Exploring the territorial politics of welfare", en N. McEwen y L. Moreno (eds.), The Territorial Politics of Welfare. Nueva York: Routledge.

Moreno, L. y Palier B. (2005), “The Europeanization of Welfare: Paradigm shifts and social policy reforms", en P. Taylor-Gooby, ed., Ideas and Welfare State Reform in Western Europe. Nueva York: Palgrave Macmillan.

Moreno, L. y Ziglio E. (1992), “Evaluación y maximización de políticas sociales”, en L. Moreno y M. Pérez-Yruela, comps., Política Social y Estado del Bienestar. Madrid: Ministerio de Asuntos Sociales.

Munchau, W. (2005), "Why social models are irrelevant", Financial Times (23 de octubre de 2005).

Palier, B. (2001), “Beyond retrenchment", Center for European Studies Working Paper Series, Harvard University, <http://www.ces.fas.harvard.edu/publications/ docs/pdfs/palier.pdf> (consulta: 30 noviembre 2008).

Potůček, M. (2006), "Welfare State Transformations in Central and Eastern Europe”. Ponencia presentada en el I Seminario Internacional de Política Social, "Regímenes de bienestar en transición”, 11 septiembre 2006, Universidad de Guadalajara (México).

Salais, R. (2006) "Reforming the European Social Model and the politics of indicator: from the unemployment rate to the employment rate in the European Employment Strategy", en M. Jepsen y A. Serrano Pascual, ed., Unwrapping the European Social Model. Bristol: Polity Press.

Salais, R., Baverez, N. y Reynaud, B. (1986) L'invention du chômage. Histoire et transformation d'une catégorie en France des années 1890 aux années 1980. París: Presses Universitaires de France.

Sapir, A. (2005), "Globalisation and the Reform of European Social Models", Bruselas: Bruegel Policy Brief <http://www.bruegel.org/doc_pdf_120> (consulta: 26 mayo 2008).

Shackleton, L. (2006), “The European Social Model: past its sell-by date”, Fundación Rafael del Pino, Madrid. <http://www.fundacionrafaeldelpino.es/inicio.asp> (consulta: 26 mayo 2008).

Scharpf, F. (2002), "The European Social Model: Coping with the Challenges of Diversity", Journal of Common Market Studies, 40 (1): 649-669.

Schram, S. (2000), "The medicalisation of welfare", Social Text 62,18 (1): 81-107.

Serrano Pascual, A., ed. (2004), Are activation policies converging in Europe? Bruselas: ETUI. 
- 2008) "Is the Open Method of Coordination a Provider of Political Tools to Promote Gender Mainstreaming?", en V. Beveridge y S. Velluti, Gender and the Open Method of Coordination, pp. 169-191, Hampshire: Ashgate.

Serrano Pascual, A., Crespo, E. (2007), "The Government of Activation Policies by EU Institutions”, International Journal of Sociology and Social Policy, 27 (9/10): 376387.

Serrano Pascual, A. y Magnusson, L. (2007) (eds.), Reshaping welfare states and activation regimes in Europe. Bruselas: Peter Lang.

Topalov, C. (1994), La naissance du chômeur 1880-1910. París: Albin Michel.

Trubeck, D. y J. Mosher (2003), "New Governance, EU Employment Policy and the European Social Model”, en J. Zeitlin y D.Trubeck, eds., Governing Work and Welfare in the New Economy: European and American Experiments. Oxford: Oxford University Press.

Van Oorschot, W. (2006), "Making the difference in social Europe: deservingness perceptions among citizens of European welfare status", Journal of European Social Policy 16 (1): 23-42.

Walters, W. y Haahr, J.H. (2005), Governing Europe. Discourse, governmentality and European Integration. Nueva York: Routledge. 